\title{
Light modulation in phoxonic nanocavities
}

\author{
N. Papanikolaou ${ }^{\mathrm{a}, *}$, I.E. Psarobas ${ }^{\mathrm{a}, \mathrm{b}}$, N. Stefanou ${ }^{\mathrm{b}}$, B. Djafari-Rouhanic ${ }^{\mathrm{c}}$ \\ B. Bonello ${ }^{\mathrm{d}}$, V. Laude ${ }^{\mathrm{e}}$ \\ ${ }^{a}$ Institute of Microelectronics, NCSR "Demokritos,"GR-153 10 Athens, Greece \\ ${ }^{b}$ Section of Solid State Physics, University of Athens, Panepistimioupolis, GR-157 84 \\ Athens, Greece \\ ${ }^{c}$ Institut d'Électronique, de Microélectronique et de Nanotechnologie, Université de Lille \\ 1, 59655 Villeneuve d'Ascq, France \\ ${ }^{d}$ Institut des NanoSciences de Paris, CNRS (UMR 7588), Université Pierre et Marie \\ Curie, 140 rue de Lourmel, 75015 Paris, France \\ 'Institut FEMTO-ST, Université de Franche Comté, CNRS, ENSMM, Besançon, France
}

\begin{abstract}
We report on the occurrence of strong nonlinear acousto-optic interactions in phoxonic structures, that support, simultaneously, acoustic and optical localized resonant modes, under the influence of acoustic losses. Deploying a detailed theoretical investigation of the acousto-optic coupling in the specific case of a one-dimensional phoxonic cavity, realized by homogeneous $\mathrm{SiO}_{2}$ and $\mathrm{Si}$ layers, we demonstrate the possibility for an enhanced modulation of light with sound through multi-phonon exchange mechanisms. A full electrodynamic and elastodynamic multiple scattering approach is employed to describe the optical and acoustic modes, and to account for their mutual interaction and the underlying effects both in time and frequency domains. In particular, we discuss the influence of hypersonic attenuation on the acousto-optic interaction by considering typical acoustic losses in the $\mathrm{GHz}$ regime.
\end{abstract}

Keywords:

Phoxonic Crystals, Acousto-optic Interaction, Inelastic Light Scattering, Hypersonic Attenuation, Nonlineal Effects

The acousto-optic (AO) interaction has been widely used to process light

\footnotetext{
*Corresponding author

Email address: N.Papanikolaou@imel.demokritos.gr (N. Papanikolaou)
} 
signals in homogeneous materials for years [1]. Moreover periodic multilayers, with or without defects, provide further possibilities for tailoring the $\mathrm{AO}$ effect and allow one to observe new, interesting, and potentially useful physical phenomena $[2,3]$. On the experimental side, ultrafast acoustics techniques $[4,5]$ can probe in a straightforward and controllable manner the $\mathrm{AO}$ interaction in nanostructures $[6,7]$ by monitoring the transmission of light perturbed by a picosecond strain pulse [8]. Such an approach has gained interest the last few years, either for identifying collective acoustic modes that could lead to light modulation in photonic crystals $[6,9]$ or, to obtain ultrafast control of optical resonances in nanostructures $[3,6]$.

Potential candidates for exploring the AO interaction between localized photons and phonons in the same region of space are structures identified as dual spectral gap materials [10], also termed phoxonic crystals $[11,12]$. These structures, because of their dual gap behavior, can be used to design phoxonic cavities that support both photonic and phononic localized modes. Coupling between such modes was anticipated in the case of a one-dimensional (1D) hypersonic phononic crystal [13], while strong Raman scattering was demonstrated in the case of a planar phonon cavity embedded within an optical nanocavity realized by a semiconductor heterostructure [14]. In a recent theoretical investigation [2] we demonstrated the occurrence of enhanced AO interactions in a model phoxonic resonant cavity realized by Bragg mirrors consisting of homogeneous $\mathrm{SiO}_{2}$ and $\mathrm{Si}$ multilayers. We assumed a $1 \mathrm{D}$ phoxonic crystal consisting of a periodic series of units described as $\left(\mathrm{SiO}_{2}-\mathrm{Si}\right)^{N}$ and $\mathrm{a} \mathrm{SiO}_{2}$ layer of different width in the middle followed by the exact mirror series $\left(\mathrm{Si}-\mathrm{SiO}_{2}\right)^{N}$ of the same units stacked along the $z$ direction. In particular, the number of units were chosen to be $6(N=6)$ and each unit had a width $a$, where the widths of the $\mathrm{SiO}_{2}$ and $\mathrm{Si}$ layers were $2 a / 3$ and $a / 3$, respectively, while the defect $\mathrm{SiO}_{2}$ layer had a width $2 a$ [see Fig. 1(b)]. The relevant material properties for $\mathrm{SiO}_{2}(\mathrm{Si})$ were characterized by the index of refraction $n=1.46$ (3.46), photoelastic coefficient $p_{12}=0.27$ (0.01), mass density $\rho=2.20(2.33) \mathrm{gr} / \mathrm{cm}^{3}$ and longitudinal sound velocity $c_{l}=5970$ (8430) $\mathrm{m} / \mathrm{s}$. We have analyzed the time evolution of the scattered optical field when the structure is excited by an acoustic wave and pointed out the differences in the AO interaction between resonant and nonresonant modes. At the acoustic resonance, we provided evidence for strong nonlinear AO effects in the phoxonic cavity leading to efficient enhanced modulation of light with sound through multi-phonon exchange mechanisms.

Nevertheless, in an actual experiment, wave damping, which arises from 
either material absorption or fabrication imperfections by transferring energy from the coherent beams to other degrees of freedom, should be taken into account. While optical absorption in $\mathrm{SiO}_{2}$ and $\mathrm{Si}$ at infrared and longer wavelengths is generally small, attenuation at hypersonic frequencies may be rather strong $[15,16]$ and finite phonon lifetime effects can significantly downgrade the multi-phonon exchange mechanism in the case of very-high$Q$ acoustic resonances. In this respect, acoustic losses should be carefully considered in the design of functional phoxonic nanocavities. The present work, appears as a follow-up investigation of how hypersonic attenuation moderates the strong $\mathrm{AO}$ interactions of a specific realization of a phoxonic nanocavity.

Picosecond ultrasonics measurements suggest a propagation length of the order of 100 microns for $\mathrm{SiO}_{2}[15,16]$ around $10 \mathrm{GHz}$, following an $\Omega^{2}$ dependence of the attenuation coefficient. For different types of $\mathrm{SiO}_{2}$ the hypersonic attenuation can be somewhat different but in any case of the same order of magnitude. On the other hand, the attenuation in $\mathrm{Si}$ is well below that in $\mathrm{SiO}_{2}$ [16]. Therefore, if interface diffusion arising from fabrication imperfections is not very strong, the propagation length is not a major limitation in $\mathrm{SiO}_{2}$-Si nanostructures around $10 \mathrm{GHz}$. Ultrasonic losses can be accounted for through complex propagation velocities $[17,18]$. We assume $c_{l}(1-0.01 i)$ for both materials, which is an overestimate, to simulate a realistic structure with a lattice constant of $300 \mathrm{~nm}$, i.e., a width of $200 \mathrm{~nm}(100 \mathrm{~nm})$ for the individual $\mathrm{SiO}_{2}(\mathrm{Si})$ layers and a width of $600 \mathrm{~nm}$ for the defect $\mathrm{SiO}_{2}$ layer.

Our calculations are carried out by the layer-multiple-scattering method, which is well documented for both elastodynamics [19] and electrodynamics [20]. This powerful tool describes accurately the acoustic and the optical response of composite structures made of a number of different layers having the same $2 \mathrm{D}$ periodicity in the $x-y$ plane (parallel to the layers). The advantage of such an approach is that it does not require periodicity in the $z$ direction (perpendicular to the layers). In our case, we deal with the simple situation where all layers are homogeneous. We choose to inject the structure with a compressional acoustic wave, at normal incidence. The corresponding hypersonic transmission spectrum is presented in Fig. 1(a) where both lossless and attenuated cases are considered. The localized phononic defect mode appears at $f=\Omega / 2 \pi=10.86 \mathrm{GHz}$. The acoustic displacement input level $u_{0}$ produces the amplitude profiles throughout the structure presented in Fig. 1(b). It can be seen that the attenuated acoustic profile is much lower than that in the lossless case. 
The photonic response of the structure exhibits a frequency gap as well, with a high- $Q$ resonance $(Q=3100)$ inside the gap at a wavelength $\lambda=$ $1555.18 \mathrm{~nm}$, as shown in Fig. 2. The acoustic wave field induces a quasi-static perturbation on the electromagnetic (EM) field by means of two distinct contributions via the AO interaction [8]. One is the interface (size) contribution due to the displacement of the boundaries of each layer, while the other contribution comes from the bulk AO effect, responsible for the inhomogeneous modulation of the dielectric function due to the spatial and temporal evolution of the strain field. We note that the choice of a resonant compressional acoustic feedback has been made because of the more pronounced perturbation of the AO interaction in the optical parameters of the system. The overall spectral features and the position of the gap do not change much as time evolves, whereas the position of the optical defect mode oscillates with a maximum wavelength shift $\Delta \lambda \simeq 0.5 \mathrm{~nm}$, at the driving frequency of the attenuated resonant acoustic excitation (see Fig. 2). We note that the resonant lossless acoustic mode induces an optical wavelength shift of $1.6 \mathrm{~nm}$. The modulation of the optical defect mode by the acoustic excitation can be understood as follows. Assuming that the acoustic wave does not affect drastically the structure under consideration so that both photonic band gap and defect mode are maintained, the induced periodic variation of the refractive index and thickness of the defect layer results in a periodic oscillation of the position of the optical defect mode in the gap with the same period. If both optical and acoustic defect modes are involved, the corresponding photonphonon interaction is enhanced because of the simultaneous concentration of the respective fields for a long time period in the defect region. In the wave picture this is manifested as a larger-amplitude oscillation of the position of the optical resonance. Correspondingly, in the particle picture, one expects strong inelastic light scattering with considerable probabilities for absorption and emission of many phonons by the photon [2]. In the presence of ultrasonic attenuation these effects are considerably reduced because of the shorter lifetime of the acoustic mode.

We now assume an EM wave at the optical resonance wavelength, incident normally on the phoxonic structure, which is continuously excited by the resonant compressional acoustic wave also propagating along the $z$ direction. While hypersonic attenuation is on, we display the calculated amplitude of the reflected electric field in the upper panel of Fig. 3 together with the reflected field in the lossless case, induced by the acoustic wave at the resonance frequency. We choose the reflected electric field, because it practically carries 
only the AO interaction terms, since in the unperturbed situation the optical wavelength corresponds to a transmission resonance. We observe sharp structures in the amplitude of the reflected field $E_{r f}(t)$ when the oscillating optical resonance passes through the observation wavelength. This temporal variation is also reflected in the corresponding Fourier spectrum (lower panel of Fig. 3) where, in addition to the strong first harmonics $(n= \pm 1)$, there are also significant higher-order terms. Similar results are also obtained if we consider the transmitted, instead of the reflected, field. The scattering process is nonlinear in the $\mathrm{AO}$ interaction which, in the particle picture, implies strong probability amplitudes for multi-phonon absorption and emission processes. Obviously the effect is somewhat diminished, as compared to the lossless acoustic case, but still the assumption of a strong inelastic light scattering process is valid [2]. We also note that there is a small elasticscattering component, which should be ascribed to multi-phonon processes with simultaneous absorption and emission of the same number of phonons. Finally, as expected, at an off-resonance acoustic frequency of $9.15 \mathrm{GHz}$, both real and imaginary parts of $E_{r f}$ have a smooth sinusoidal-like variation with time (not shown here). The corresponding Fourier spectrum is essentially dominated by the first-order term while all higher-order terms are at least one order of magnitude smaller. This is the behavior expected in a usual pump-probe or Brillouin/Raman scattering experiment where single-phonon processes are involved and expansion up to first order is sufficient (Stokes and anti-Stokes components) $[8,21]$. The situation is the same at any wavelength off the optical resonance with the acoustic excitation being either off or at resonance. On the contrary, if the optical and acoustic modes involved in the inelastic light scattering process are both simultaneously localized in the same region (defect region) for a long time period, strong interaction takes place. These results conform exactly with the conclusions drawn in Ref. [2], where no hypersonic attenuation was considered.

In conclusion, we have demonstrated that, even in the presence of hypersonic attenuation in the phoxonic nanostructure under study, localized phonons can still trigger nonlinear AO interactions, beyond single phonon absorption and emission processes, leading to strong modulation of light with sound. 


\section{Acknowledgments}

This work was financially supported by the FET-Open project TAILPHOX (Grant Number 233883).

\section{References}

[1] A. Yariv and P. Yeh, Optical waves in Crystals, John Wiley and Sons, New York 1984.

[2] I. E. Psarobas, N. Papanikolaou, N. Stefanou, B. Djafari-Rouhani, B. Bonello, and V. Laude, Phys. Rev. B 82 (2010), 174303.

[3] X.S. Qian, J.P. Li, M.H. Lu, and Y.F. Chen, J. Appl. Phys. 106 (2009), 043107.

[4] B. Bonello, B. Perrin, and C. Rossignol, J. Appl. Phys. 83 (1998), 30813088.

[5] J.F. Robillard, A. Devos, I. Roch-Jeune, and P.A. Mante, Phys. Rev. B 78 (2008), 064302.

[6] T. Berstermann, C. Brüggemann, M. Bombeck, A.V. Akimov, D.R. Yakovlev, C. Kruse, D. Hommel, and M. Bayer, Phys. Rev. B 81 (2010), 085316.

[7] P. M. Walker, J.S. Sharp, A.V. Akimov, and A.J. Kent, Appl. Phys. Lett. 97 (2010), 073106.

[8] O. Matsuda, and O.B. Wright, J. Opt. Soc. Am. B 19 (2002), 3028-3041.

[9] W.Y. Huang, W. Qian, and M.A. El-Sayed, Adv. Mater. 20 (2008), 733-737.

[10] T. Gorishnyy, M. Maldovan, C. Ullal, and E.L. Thomas, Physics World 18 (2005), 24.

[11] S. Sadat-Saleh, S. Benchabane, F.I. Baida, M.P. Bernal, and V. Laude, J. Appl. Phys. 106 (2009), 074912.

[12] N. Papanikolaou, I.E. Psarobas, and N. Stefanou, Appl. Phys. Lett. 96 (2010), 231917. 
[13] N. Gomopoulos, D. Maschke, C.Y. Koh, E.L. Thomas, W. Tremel, H. J. Butt, and G. Fytas, Nano Lett. 10 (2010), 980-984.

[14] M. Trigo, A. Bruchhausen, A. Fainstein, B. Jusserand, and V. ThierryMieg, Phys. Rev. Lett. 89 (2002), 227402.

[15] P. Emery and A. Devos, Appl. Phys. Lett. 89 (2006), 191904.

[16] J.Y. Duquesne and B. Perrin, Phys. Rev. B 68 (2003) 134205.

[17] I.E. Psarobas, Phys. Rev. B 64 (2001), 012303.

[18] R. Sainidou, N. Stefanou, I.E. Psarobas, and A. Modinos, Phys. Rev. B 66 (2002), 024303.

[19] R. Sainidou, N. Stefanou, I.E. Psarobas, and A. Modinos, Comput. Phys. Commun. 166 (2005), 197-240.

[20] N. Stefanou, V. Yannopapas, and A. Modinos, Comput. Phys. Commun. 132 (2000), 189-196.

[21] J. He, B. Djafari-Rouhani, and J. Sapriel, Phys. Rev. B 37 (1988), 40864098 . 

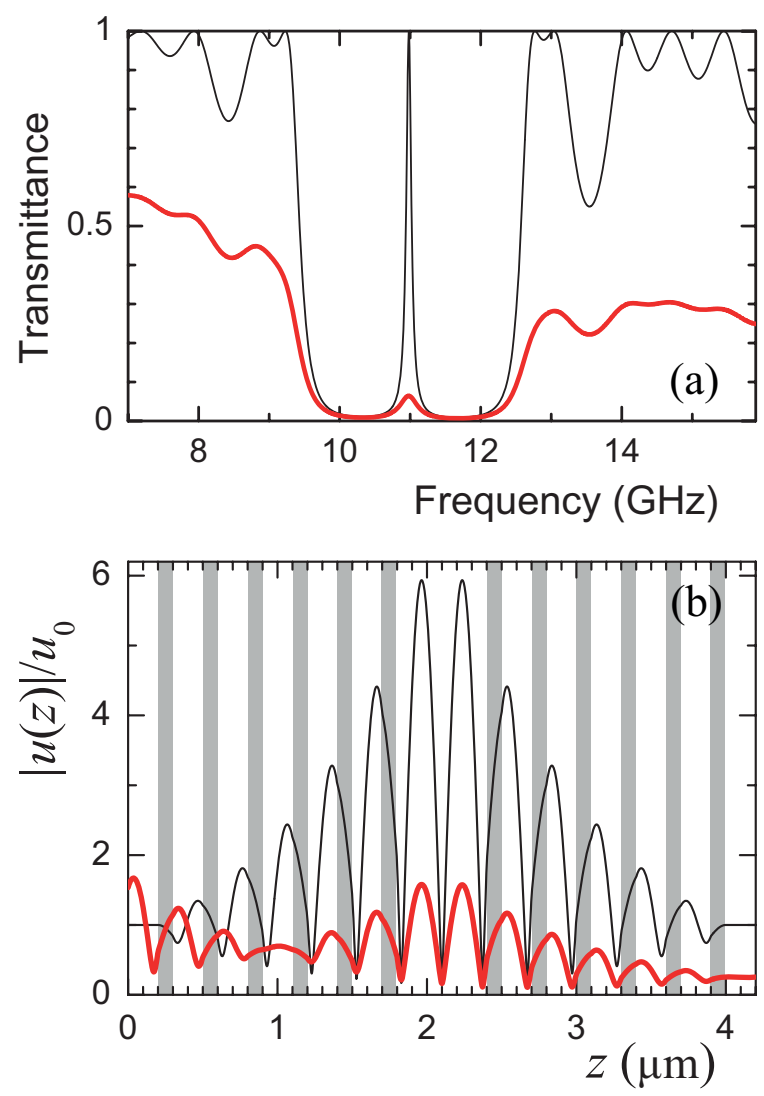

Figure 1: (a) Phononic transmission spectrum of a compressional acoustic wave normally incident on the phoxonic crystal under consideration without (thin line) and with (thick line) losses. (b) Displacement amplitude profile, normalized to the input displacement level $u_{0}$, at the acoustic resonance without losses (thin line) and with hypersonic attenuation taken into account (thick line). White and gray stripes in the background show the regions of $\mathrm{SiO}_{2}$ and $\mathrm{Si}$, respectively. 

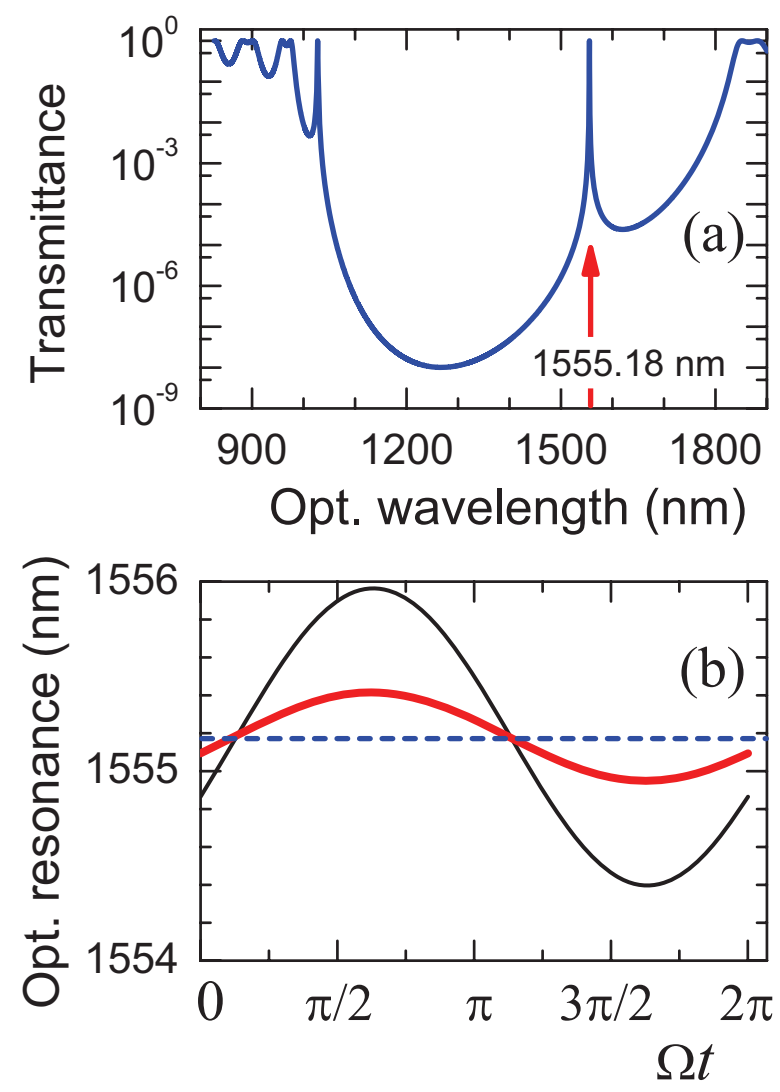

Figure 2: (a) Optical transmission spectrum of the phoxonic crystal under study, at normal incidence, without the AO interaction. The optical resonance appears at $1555.18 \mathrm{~nm}$. (b) Oscillatory behavior of the optical resonance as a function of time for the attenuated acoustic excitation at resonance (thick line) compared to the lossless acoustic excitation (thin line). The input displacement level is $u_{0}=0.1 \mathrm{~nm}$ in both cases. 

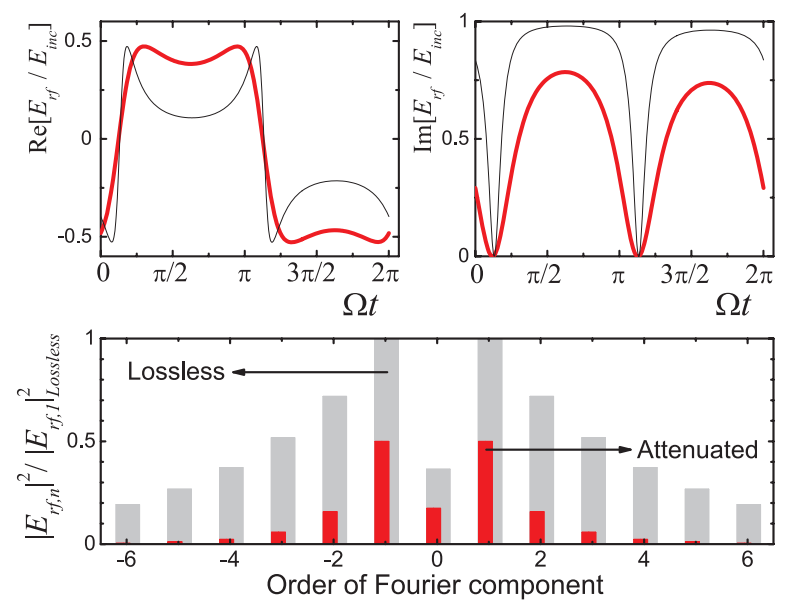

Figure 3: Optical reflection through the given phoxonic structure under a continuous acoustic excitation of input level $u_{0}=0.1 \mathrm{~nm}$, for normally incident light at the resonance wavelength with electric field amplitude $E_{i n c}$. Time variation of the perturbed reflected optical field (upper diagrams) and intensities associated with the corresponding Fourier components (lower diagram). The perturbation refers to the AO interaction with the acoustic excitation at the resonance frequency of $10.86 \mathrm{GHz}$ with hypersonic attenuation. For comparison, the corresponding lossless acoustic excitation graphs are shown as thin lines in the upper diagram and as grey background bars in the lower one. The Fourier components on the lower panel are normalized with respect to the first order component with a resonant acoustic excitation without losses. 